\title{
Testing Linear-Invariant Function Isomorphism
}

\author{
Karl Wimmer ${ }^{1 \star}$ and Yuichi Yoshida ${ }^{2 \star \star}$ \\ 1 Duquesne University \\ 2 National Institute of Informatics and Preferred Infrastructure, Inc. \\ wimmerk@duq.edu, yyoshida@nii.ac.jp
}

\begin{abstract}
A function $f: \mathbb{F}_{2}^{n} \rightarrow\{-1,1\}$ is called linear-isomorphic to $g$ if $f=g \circ A$ for some non-singular matrix $A$. In the $g$-isomorphism problem, we want a randomized algorithm that distinguishes whether an input function $f$ is linear-isomorphic to $g$ or far from being so.

We show that the query complexity to test $g$-isomorphism is essentially determined by the spectral norm of $g$. That is, if $g$ is close to having spectral norm $s$, then we can test $g$-isomorphism with poly $(s)$ queries, and if $g$ is far from having spectral norm $s$, then we cannot test $g$-isomorphism with $o(\log s)$ queries. The upper bound is almost tight since there is indeed a function $g$ close to having spectral norm $s$ whereas testing $g$ isomorphism requires $\Omega(s)$ queries. As far as we know, our result is the first characterization of this type for functions. Our upper bound is essentially the Kushilevitz-Mansour learning algorithm, modified for use in the implicit setting.

Exploiting our upper bound, we show that any property is testable if it can be well-approximated by functions with small spectral norm. We also extend our algorithm to the setting where $A$ is allowed to be singular.
\end{abstract}

\section{Introduction}

In this paper, we are concerned with property testing of Boolean functions. We say that two Boolean functions $f$ and $f^{\prime}: \mathbb{F}_{2}^{n} \rightarrow\{-1,1\}$ are $\epsilon$-far if the distance $\operatorname{dist}\left(f, f^{\prime}\right):=\left|\left\{x \in \mathbb{F}_{2}^{n} \mid f(x) \neq f^{\prime}(x)\right\}\right| / 2^{n}$ between $f$ and $f^{\prime}$ is at least $\epsilon$, and we call them $\epsilon$-close otherwise. We say that a function $f: \mathbb{F}_{2}^{n} \rightarrow\{-1,1\}$ is $\epsilon$-far from a property $P$ if $f$ is $\epsilon$-far from every function $f^{\prime}$ satisfying $P$ and $\epsilon$-close otherwise. A randomized query algorithm $A$ with oracle access to an unknown function $f: \mathbb{F}_{2}^{n} \rightarrow\{-1,1\}$ is called an $\epsilon$-tester for $P$ if it accepts with probability at least $\frac{2}{3}$ when $f$ satisfies $P$ and rejects with probability at least $\frac{2}{3}$ when $f$ is $\epsilon$-far from $P$. The efficiency of a tester is measured by its query complexity, that is,

\footnotetext{
* Supported by NSF award CCF-1117079.

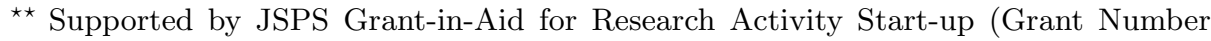
24800082), MEXT Grant-in-Aid for Scientific Research on Innovative Areas (Grant Number 24106001), and JST, ERATO, Kawarabayashi Large Graph Project.
} 
the number of queries made to the oracle. Here, we are chiefly interested in testers that make a number of queries independent of $n$ (although the number of queries can depend on some complexity measure); we refer to such testers as making a constant number of queries.

Property testing was first introduced by Rubinfeld and Sudan [1] to test algebraic properties of a function. Goldreich, Goldwasser, and Ron [2] extended the scope of this definition to graphs and other combinatorial objects. Since then, the field of property testing has been very active. For an overview of recent developments, we refer the reader to the surveys $[3$, 4] and the book [5].

A notable achievement in the field of property testing is the complete characterization of graph properties that are testable with a constant number of queries [6]. An ambitious open problem is obtaining a similar characterization for properties of Boolean functions. Recently there has been a lot of progress on the restriction of this question to linear-invariant properties [7-9]. A property $P$ of Boolean functions $\mathbb{F}_{2}^{n} \rightarrow\{-1,1\}$ is called linear-invariant if a function $f$ satisfies $P$, then $f \circ A$ is also in $P$ for any square matrix $A$, where $(f \circ A)(x):=f(A x)$ for any $x \in \mathbb{F}_{2}^{n}$. For a thorough discussion of linear-invariant properties, we refer the reader to Sudan's survey on the subject [10].

Despite much effort, we are still far from reaching a characterization of constant-query testable linear-invariant properties with two-sided error. In this paper, we consider function isomorphism testing, which in some sense is the simplest possible linear-invariant property. Given a Boolean function $g$, the $g$-isomorphism testing problem is to determine whether a function $f$ is isomorphic to $g$, that is, whether $f=g \circ A$ for some nonsingular ${ }^{3}$ matrix $A$ or far from being so. A natural goal, and the focus of this paper, is to characterize the set of functions for which isomorphism testing can be done with a constant number of queries.

Our first contribution is revealing a parameter of functions that makes isomorphism testing easy. To state our result precisely, let us introduce several definitions about Fourier analysis. Using characteristic functions $\chi_{\alpha}(x):=(-1)^{\langle\alpha, x\rangle}=(-1)^{\sum_{i=1}^{n} \alpha_{i} x_{i}}$, every Boolean function $f: \mathbb{F}_{2}^{n} \rightarrow$ $\{-1,1\}$ has a unique representation as $f(x)=\sum_{\alpha \in \mathbb{F}_{2}^{n}} \widehat{f}(\alpha) \chi_{\alpha}(x)$. The coefficients $\widehat{f}(\alpha)$ are called the Fourier coefficients of $f$. Then, the spec-

\footnotetext{
3 The definition of linear invariance allows for using matrices $A$ that could be singular. However, this definition does not induce an equivalence relation on the set of all Boolean functions. Thus, we restrict $A$ to be non-singular to focus on isomorphism. As a corollary of our isomorphism work, we can handle the full linear-invariant case.
} 
tral norm of $f$ is defined as $\widehat{\|}_{f \|_{1}}:=\sum_{\alpha \in \mathbb{F}_{2}^{n}}|\widehat{f}(\alpha)|$. We show that $\widehat{\|}_{\|} \widehat{\|}_{1}$ essentially determines the hardness of testing $g$-isomorphism:

Theorem 1 (Main theorem). Suppose that $g: \mathbb{F}_{2}^{n} \rightarrow\{-1,1\}$ is $\frac{\epsilon}{3}$ close to having $\widehat{\|} g \widehat{\|}_{1} \leq s$. Then, we can $\epsilon$-test $g$-isomorphism with query complexity poly $\left(s, \frac{1}{\epsilon}\right)$ queries.

Indeed, Theorem 1 is proved by giving a tolerant tester for the property of having spectral norm at most $s$. That is, it accepts every function $\frac{\epsilon}{2}$-close to having spectral norm at most $s$ with probability at least $\frac{2}{3}$ whereas it rejects every function $\epsilon$-far from having spectral norm at most $s$.

In contrast to Theorem 1, if a function $g$ is far from having small spectral norm, then $g$-isomorphism becomes hard to test:

Theorem 2. Suppose that $g: \mathbb{F}_{2}^{n} \rightarrow\{-1,1\}$ is $\epsilon$-far from having $\widehat{\|} g \widehat{\|}_{1} \leq$ $s$. Then, $\epsilon$-testing g-isomorphism requires $\Omega(\log s)$ queries.

We note that Fischer obtained a similar result for graph isomorphism testing in the dense graph model [11] using complexity of graphs instead of spectral norm. Here, the complexity of a graph is the size of a partition needed to express the graph, that is, an edge exists between two parts in the partition if and only if all vertices between the two parts are adjacent. His result has been used as an important tool to obtain the complete characterization of constant-query testable graph properties $[6,12]$. Thus, we believe that our result will be also useful to obtain a characterization for function properties.

Theorem 1 is close to tight as is shown in the following theorem.

Theorem 3. For any $s>0$, there exists a function $g$ with $\widehat{\|}_{\|} \widehat{\|}_{1} \leq s$ such that testing g-isomorphism requires $\Omega(s)$ queries.

For a function $f: \mathbb{F}_{2}^{n} \rightarrow\{-1,1\}, \operatorname{Spec}(f):=\left\{\alpha \in \mathbb{F}_{2}^{n} \mid \widehat{f}(\alpha) \neq 0\right\}$ denotes the spectrum of $f$. A function $f$ is called $r$-sparse if $|\operatorname{Spec}(f)| \leq$ $r$ and having Fourier dimension $k$ if $\operatorname{Spec}(f)$ lies in an $k$-dimensional subspace of $\mathbb{F}_{2}^{n}$. It is not hard to show that an $r$-sparse function has spectral norm at most $\sqrt{r}$ and an $k$-dimensional function has spectral norm at most $2^{k / 2}$. Thus, as corollaries of Theorem 1 , we can test $g$ isomorphism with a constant number of queries when $g$ is close to being $r$-sparse for constant $r$ or $k$-dimensional for constant $k$. More generally, we can test any property that can be approximated by a set of functions such that each function in the set has small spectral norm.

Theorem 4. Let $P$ be a linear-invariant property. Suppose that every

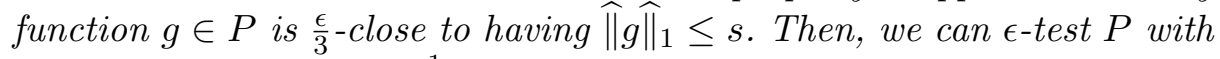
query complexity $\operatorname{poly}\left(s, \frac{1}{\epsilon}\right)$. 
For example, with this theorem, we can test whether an input function is a small Boolean circuit in which each input is made by the parity of variables. It is shown that $r$-sparsity and $k$-dimensionality are constantquery testable in [13]. As a corollary of Theorem 4, these properties are tolerantly constant-query testable.

Further, we can extend Theorem 1 to the case where we alter the definition of linear isomorphism to allow for singular linear transformations.

Proof sketch of Theorem 1.1 We prove our main theorem in a similar manner to the implicit learning method used in [14] and [13]. Similarly to [13], our algorithm finds all the large Fourier coefficients of the unknown function $f$ using an implicit version of the Goldreich-Levin algorithm [15]; we call our algorithm Implicit Sieve. Given a set of vectors $\mathcal{M}$, it outputs $\left\{\left\langle\chi_{\alpha_{1}}(x), \ldots, \chi_{\alpha_{k}}(x), f(x)\right\rangle \mid x \in \mathcal{M}\right\}$, where $\alpha_{i}$ 's are (implicitly) chosen to cover large Fourier coefficients. The approach in [13] works when $f$ is $k$-dimensional; our algorithm Implicit Sieve gives correct output with no assumptions about $f$.

Once Implicit Sieve is run, rather than checking consistency as in [14] and [13], our algorithm produces a sparse polynomial that approximates $f$. This can be done by estimating $\widehat{f}\left(\alpha_{i}\right)$ from the output of Implicit Sieve. To check isomorphism to a given function $g$ (or even multiple functions), the algorithm tries to "fit" the Fourier mass of $f$ into the Fourier mass of $g$. We note that checking this "fit" requires no further queries. If the spectral norm of $g$ is small, then this algorithm will succeed, and in fact does so in a tolerant manner. We note the strong connection of learning functions with small spectral norm due to Kushilevitz and Mansour [16].

Finally, we show that tolerant isomorphism testing implies that we can test properties that are well-approximated by functions with small spectral norm.

Related Work. Another natural setting of function isomorphism is that we regard two functions $f, f^{\prime}: \mathbb{F}_{2}^{n} \rightarrow\{-1,1\}$ are isomorphic if they are the same up to relabeling of bits. In this setting, it is known that $g$-isomorphism is constant-query testable if $g$ is a $k$-junta for constant $k$ [17] or a symmetric function, and the result was extended to $(n-k)$ symmetric functions $[18,19]$. Here, a function is called a $k$-junta if it only depends on $k$ bits in the input string and called an $(n-k)$-symmetric function if there exist some $k$ bits such that it becomes a symmetric function for every fixed setting of the $k$ bits. It is also conjectured that the parameter $k$ above is the parameter that determines the difficulty of 
$g$-isomorphism in this setting as the spectral norm is the parameter in the linear isomorphism setting $[18,19]$.

In recent work, Grigorescu et al. [20] consider extremal examples of lower bounds for testing linear isomorphism in the same sense considered here. In that work, the authors show that there is a function that requires $\Omega\left(n^{2}\right)$ queries for testing linear isomorphism. The spectral norm of this function is exponential in $n$, so this example is quite far away from what we consider here, since we consider functions of "constant" complexity.

Very recently, [21] gave a characterization of affine-invariant properties that are constant-query testable with one-sided error. Their work does not derive our result since having small spectral norm and satisfying the condition they gave are incomparable. Also, we consider two-sided error testability of linear-invariant properties instead of one-sided error testability of affine-invariant properties.

In graph property testing, there are two major models, called the dense graph model and the bounded-degree model. In the dense graph model, we have access to the adjacency matrix and a graph $G$ is called $\epsilon$ far from a property $P$ if we must add or remove at least $\epsilon n^{2}$ edges to make $G$ satisfy $P$. As we already mentioned, [11] showed that the difficulty of testing $H$-isomorphism is determined by the complexity of $H$. The gap between its upper and lower bounds on query complexity in terms of the graph complexity was improved in [19]. [22] showed that the query complexity to test $H$-isomorphism for general $H$ is $\widetilde{\Theta}(\sqrt{n})$ with two-sided error and $\widetilde{\Theta}(n)$ with one-sided error. In the bounded-degree model with a degree bound $d$, we only deal with graphs with maximum degree at most $d$. We have access to the incidence list and a graph $G$ is called $\epsilon$-far from a property $P$ if we must add or remove at least $\epsilon d n$ edges to make $G$ satisfy $P$. [23] showed that we can test $H$-isomorphism in constant time when $H$ is hyperfinite, that is, for any $\epsilon>0$ we can decompose $H$ into connected components of size $s=s(\epsilon)$ by deleting $\epsilon n$ edges. A notable example of hyperfinite graphs is planar graphs. Exploiting this result, [23] showed that every hyperfinite property is constant-query testable.

Organization. In Section 2, we introduce definitions and useful facts used throughout this paper. We introduce Implicit Sieve in Section 3 and proceed to prove our main theorem in Section 4. We extend our upper bounds to the linear-invariant case using our linear isomorphism results and prove Theorem 4 in Appendix D. We prove a lower bound for testing linear isomorphism to functions of small spectral norm in Appendix E. 


\section{Preliminaries}

We use bold symbols to denote random variables. For a collection of vectors $\mathcal{A}$, we write $w t_{2}(\mathcal{A})=\sum_{\alpha \in \mathcal{A}} \widehat{f}(\alpha)^{2}$ and $w t_{4}(\mathcal{A})=\sum_{\alpha \in \mathcal{A}} \widehat{f}(\alpha)^{4}$. This notation suppresses the dependence on $f$, but it will always be clear from the context. For a subspace $H \leq \mathbb{F}_{2}^{n}$ and any vector $r \in \mathbb{F}_{2}^{n}$, the coset $r+H$ is defined as $\{\alpha+r \mid \alpha \in H\}$. Given a set of vectors $C \subseteq \mathbb{F}_{2}^{n}$, let $\mathrm{P}_{C}(f)$ be the projection function for $C ; \mathrm{P}_{C}(f)(x)=\sum_{\alpha \in C} \widehat{f}(\alpha) \chi_{\alpha}(x)$.

We show the following two lemmas in Appendix A.

Lemma 1. For a non-singular matrix $A, \widehat{f \circ A}(\alpha)=\widehat{f}\left(\left(A^{-1}\right)^{T} \alpha\right)$. In particular, $\widehat{g}(\alpha)=\widehat{f}\left(\left(A^{-1}\right)^{T} \alpha\right)$ when $f=g \circ A$.

Lemma 2. For any subspace $H$, any vector $r$, and any integer $k \geq 2$, we can estimate $\mathrm{wt}_{2}(r+H)$ and $\mathrm{wt}_{4}(r+H)$ to within $\pm \tau$ with confidence $1-\delta$ using $O\left(\frac{\log (1 / \delta)}{\tau^{2}}\right)$ queries.

\section{The Implicit Sieve}

As a first step, we give a general algorithm for implicitly accessing the Fourier coefficients of an unknown function $f$, which we call Implicit Sieve. The guarantee of the algorithm is stated in the following lemma.

Lemma 3. Given a threshold value $\theta>0$ and a set $\mathcal{M} \subseteq \mathbb{F}_{2}^{n}$ with $|\mathcal{M}|=$ $m$, there is an algorithm that uses poly $(m, 1 / \theta)$ queries and returns, with high probability, for some set $S=\left\{\alpha_{1}, \alpha_{2}, \ldots, \alpha_{k}\right\}$, a labeled set of $m$ examples of the form $\left\{\left\langle\chi_{\alpha_{1}}(x), \chi_{\alpha_{2}}(x), \ldots, \chi_{\alpha_{k}}(x), f(x)\right\rangle \mid x \in \mathcal{M}\right\}$, where

- Every vector $\alpha$ such that $|\widehat{f}(\alpha)| \geq \theta$ is in $S$.

- Every vector $\alpha \in S$ satisfies $|\widehat{f}(\alpha)| \geq \frac{1}{2} \theta$.

Due to space limitations, all the proofs are deferred to Appendix B. Note that the algorithm does not return the set $S$. The two guarantees here are similar to the guarantee given by the Goldreich-Levin Theorem [15] (see also [16]). The query complexity is independent of $f$.

We first introduce random $t$-coset structures.

Definition 1 ([13]). For an integer $t$, we define a random $t$-dimensional coset structure $(H, \mathcal{C})$ as follows: We choose vectors $\beta_{1}, \ldots, \beta_{t} \in \mathbb{F}_{2}^{n}$ independently and uniformly at random and set $H=\operatorname{span}\left\{\beta_{1}, \ldots, \beta_{t}\right\}^{\perp}$. For each $b \in \mathbb{F}_{2}^{t}$ we define the "bucket" $C(b)=\left\{\alpha \in \mathbb{F}_{2}^{n} \mid\left\langle\alpha, \beta_{i}\right\rangle=\right.$ $b_{i}$ for all $\left.i\right\}$. We take $\mathcal{C}$ to be the multiset of $C(b)$ 's, which has cardinality $2^{t}$. In a random permuted $t$-dimensional coset structure, we additionally choose a random $z \in \mathbb{F}_{2}^{t}$ and rename $C(b)$ by $C(b+z)$. 


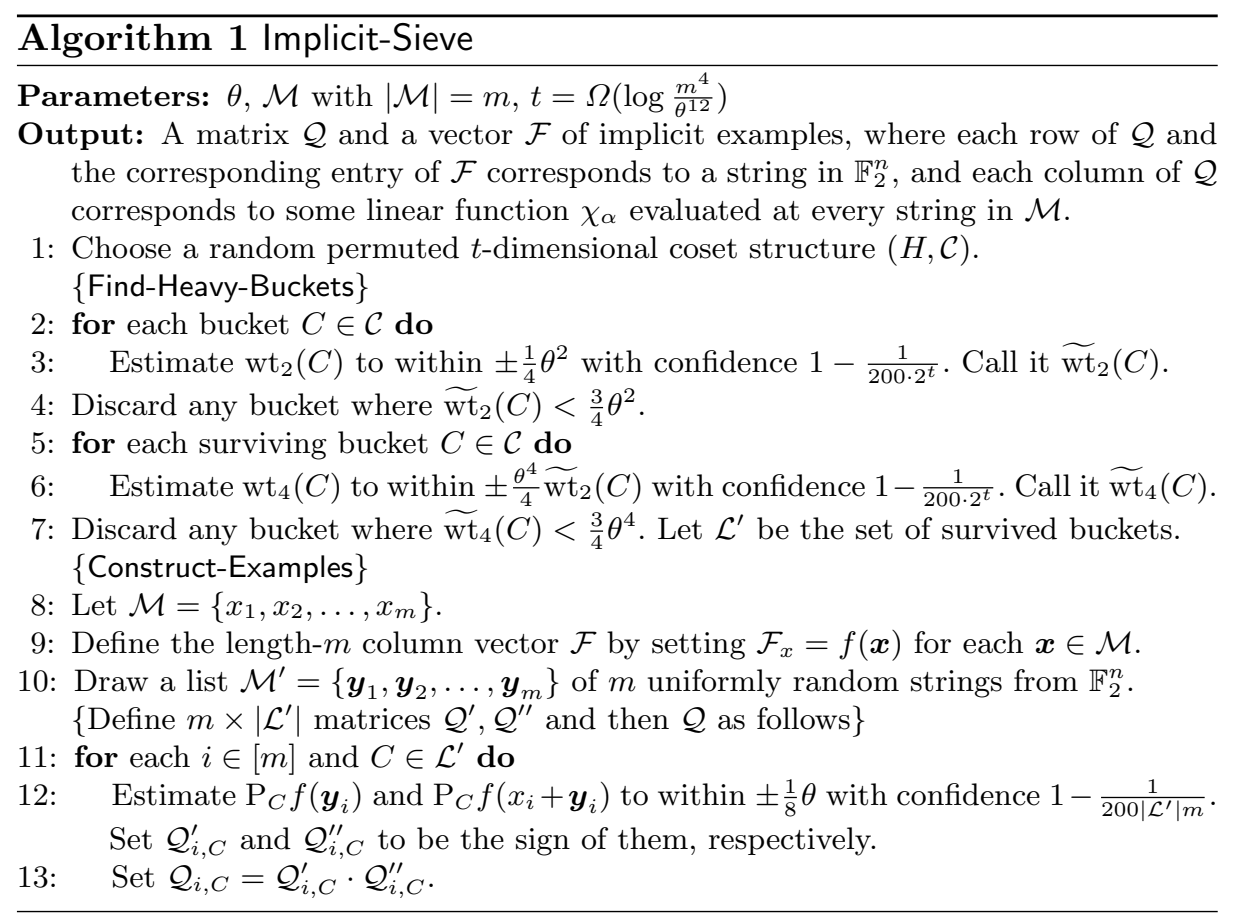

In the (unlikely) case that $\beta_{i}$ 's are linearly dependent, some of $C(b)$ 's will be cosets in $\mathbb{F}_{2}^{n} / H$ and some of them will be empty. For the empty buckets $C(b)$ we define $P_{C(b)} f$ to be identically 0 . Note that the zero vector always gets mapped to the bucket $C(0)$ in a random coset structure. To avoid technical issues caused by this fact, we adopt random permuted coset structures.

Lemma 4. Let $(H, \mathcal{C})$ be a random permuted t-dimensional coset structure, where $t \geq 2 \log \frac{16}{\theta^{4}}+\log 100$. Then for any set $S$ of vectors $\alpha$ with $|\widehat{f}(\alpha)| \geq \frac{\theta^{2}}{4}$, every vector in $S$ gets mapped into a different bucket except with probability at most $\frac{1}{100}$.

Our algorithm is given in Algorithm 1. A bucket is heavy if it contains some $\alpha \in \mathbb{F}_{2}^{n}$ with $|\widehat{f}(\alpha)| \geq \frac{\theta^{2}}{4}$. We now explain how Algorithm 1 works.

We first choose a random permuted coset structure (Step 1). Conditioning on Lemma 4, for all the heavy buckets, there is a unique Fourier coefficient of squared magnitude at least $\frac{\theta^{2}}{4}$ contained in that bucket. For any heavy bucket $C$, let $\alpha(C)$ be this unique Fourier coefficient. Then, we can show that the Fourier mass of a bucket $C$ is dominated by $\alpha(C)$ : 
Lemma 5. Suppose $t$ is such that $10 \cdot 2^{-t / 2}+2^{-t} \leq\left(\frac{\theta^{3}}{3200 m}\right)^{2}$. Assume that the condition of Lemma 4 holds. Then except with probability at most $\frac{2}{100}, \mathrm{wt}_{2}(C) \leq \widehat{f}(\alpha(C))^{2}+\left(\frac{\theta^{3}}{3200 m}\right)^{2}$ for every heavy bucket $C$.

Next, by estimating $\mathrm{wt}_{2}(C)$ and $\mathrm{wt}_{4}(C)$, we discard buckets $C \in \mathcal{C}$ that are judged to be non-heavy (Steps $2-7$ ). Let $\mathcal{L}^{\prime}$ be the set of buckets we did not discard. With high probability, we do not make any mistake and do not miss any large Fourier coefficient:

Lemma 6. Except with probability at most $\frac{1}{100}$,

- Find-Heavy-Buckets discards every bucket $C$ with $|\widehat{f}(\alpha)|<\frac{\theta^{2}}{4}$ for every $\alpha \in C$.

- Find-Heavy-Buckets does not discard any bucket $C$ with $|\widehat{f}(\alpha(C))| \geq \theta$. - $|\widehat{f}(\alpha(C))| \geq \frac{\theta}{2}$ for every $C \in \mathcal{L}^{\prime}$.

From the last property, there are at most $\frac{4}{\theta^{2}}$ many buckets in $\mathcal{L}^{\prime}$.

We proceed to analyze Construct-Examples (Steps 8-13). For each heavy bucket $C \in \mathcal{L}^{\prime}$, define $S_{C}(f)$ to be the "small projection": $S_{C}(f)(x)=$ $\sum_{\alpha \in C \backslash\{\alpha(C)\}} \widehat{f}(\alpha) \chi_{\alpha}(x)$.

Lemma 7. For each heavy bucket $C, \operatorname{Pr}_{\boldsymbol{x}}\left[\left|S_{C}(f)(\boldsymbol{x})\right| \geq \frac{1}{4} \theta\right] \leq \frac{\theta^{2}}{800 m}$.

Since $\mathrm{P}_{C}(f)(x)=\widehat{f}(\alpha(C)) \chi_{\alpha(C)}(x)+S_{C}(f)(x)$ and $|\widehat{f}(\alpha(C))|$ is much larger than $\left|S_{C}(f)(x)\right|$ from Lemmas 6 and 7 , we have that $\operatorname{sgn}\left(\widehat{f}(\alpha(C)) \chi_{\alpha}(\boldsymbol{x})\right.$ is equal to $\operatorname{sgn}\left(P_{C}(f(\boldsymbol{x}))\right.$ with high probability:

Lemma 8. For each heavy bucket $C, \operatorname{Pr}\left[\left|\mathrm{P}_{C}(f)(\boldsymbol{x})\right|<\frac{1}{4} \theta\right.$ or $\operatorname{sgn}\left(\mathrm{P}_{C}(f)(\boldsymbol{x})\right) \neq$ $\left.\operatorname{sgn}\left(\widehat{f}(\alpha(C)) \chi_{\alpha(C)}(\boldsymbol{x})\right)\right] \leq \frac{\theta^{2}}{800 \mathrm{~m}}$.

From Lemma 8 , each of the at most $2\left|\mathcal{L}^{\prime}\right| m$ estimates are correct except with probability $\frac{1}{200\left|\mathcal{L}^{\prime}\right| m}$, so these estimations add at most $\frac{1}{100}$ to the failure probability. Thus, each fixed entry of $\mathcal{Q}^{\prime}$ and $\mathcal{Q}^{\prime \prime}$ is correct except with probability at most $\frac{\theta^{2}}{800 \mathrm{~m}}$. We use basic self-correction to form the matrix $\mathcal{Q}$; a fixed entry of $\mathcal{Q}$ (a proposed value of $\chi_{\alpha(C)}$ ) is correct except with probability at most $\frac{\theta^{2}}{400 \mathrm{~m}}$. There are only $\frac{4}{\theta^{2}}$ heavy buckets at most, so except with probability at most $\frac{1}{100 m}$, a fixed row of $\mathcal{Q}$ is correct on any specific example. Thus, if we draw a set of at most $m$ (implicit) examples, the entire matrix $\mathcal{Q}$ is correct except with probability at most $\frac{1}{100}$, assuming all the previous estimates were correct. Overall, the total failure probability is at most $\frac{1}{100}+\frac{2}{100}+\frac{1}{100}+\frac{1}{100}+\frac{1}{100}=\frac{6}{100}$ as claimed; this establishes Lemma 3.

Enumerate the buckets of $\mathcal{L}^{\prime}$ as $\left\{C_{1}, C_{2}, \ldots, C_{\left|\mathcal{L}^{\prime}\right|}\right\}$. Similar to how we define $\alpha(C)$, let $\alpha\left(\mathcal{L}^{\prime}\right)=\left\{\alpha(C) \mid C \in \mathcal{L}^{\prime}\right\}$. The final matrix $\mathcal{Q}$ and vector $\mathcal{F}$ are, except with probability at most $\frac{6}{100}$, of the following form: 
$\overline{\text { Algorithm } 2 \text { Tolerant } g \text {-isomorphism testing to a function with small }}$ spectral norm

Parameters: $s, g: \mathbb{F}_{2}^{n} \rightarrow\{-1,1\}$ with $\widehat{\|}_{\|} \widehat{\|}_{1} \leq s, \epsilon$

1: Run Algorithm 1 with $\theta=\frac{\epsilon}{6 s}, m=\widetilde{O}\left(\frac{s^{2}}{\epsilon^{2}}\right)$, and $\mathcal{M}$ a uniformly random subset of $\mathbb{F}_{2}^{n}$ with $|\mathcal{M}|=m$ to get $(\mathcal{Q}, \mathcal{F})$.

2: for each $C \in \mathcal{L}^{\prime}$ do

3: $\quad$ Use $(\mathcal{Q}, \mathcal{F})$ to estimate $\widehat{f}(\alpha(C))$ within $\pm \frac{\epsilon}{3 s}$ with confidence $1-\frac{1}{100\left|\mathcal{L}^{\prime}\right|}$ and call it $\widetilde{\widehat{f}}(\alpha(C))$.

4: Set $\widetilde{\widehat{f}}(\alpha)=0$ for all $\alpha \in \mathbb{F}_{2}^{n} \backslash \alpha\left(\mathcal{L}^{\prime}\right)$.

5: Accept if there is a nonsingular linear transformation $A$ such that $\sum_{\beta \in \mathbb{F}_{2}^{n}} \widetilde{\widetilde{f \circ A}}(\beta) \widehat{g}(\beta) \geq 1-\epsilon$, and reject otherwise.

\begin{tabular}{c|cccc|c}
$(\mathcal{Q} \mid \mathcal{F})$ & $C_{1}$ & $C_{2}$ & $\ldots$ & $C_{\left|\mathcal{L}^{\prime}\right|}$ & $f(x)$ \\
\hline$x_{1}$ & $\chi_{\alpha\left(C_{1}\right)}\left(x_{1}\right)$ & $\chi_{\alpha\left(C_{2}\right)}\left(x_{1}\right)$ & $\ldots$ & $\chi_{\alpha\left(C_{\left|\mathcal{L}^{\prime}\right|}\right)}\left(x_{1}\right)$ & $f\left(x_{1}\right)$ \\
$x_{2}$ & $\chi_{\alpha\left(C_{1}\right)}\left(x_{2}\right)$ & $\chi_{\alpha\left(C_{2}\right)}\left(x_{2}\right)$ & $\ldots$ & $\chi_{\alpha\left(C_{\left|\mathcal{L}^{\prime}\right|}\right)}\left(x_{2}\right)$ & $f\left(x_{2}\right)$ \\
$\vdots$ & $\vdots$ & $\vdots$ & $\ddots$ & $\vdots$ & $\vdots$ \\
$x_{m}$ & $\chi_{\alpha\left(C_{1}\right)}\left(x_{m}\right)$ & $\chi_{\alpha\left(C_{2}\right)}\left(x_{m}\right)$ & $\ldots$ & $\chi_{\alpha\left(C_{\left|\mathcal{L}^{\prime}\right|}\right)}\left(x_{m}\right)$ & $f\left(x_{m}\right)$
\end{tabular}

where $\mathcal{M}=\left\{x_{1}, x_{2}, \ldots, x_{m}\right\}$.

The query complexity of this algorithm is dominated by the estimates in Steps 2 and 3. Since the number of buckets is $\Omega\left(\frac{m^{4}}{\theta^{12}}\right)$, we need $\widetilde{O}\left(\frac{m^{4}}{\theta^{16}}\right)$ queries in total to make one estimate to tolerance $\pm \frac{\theta^{2}}{4}$ for each bucket.

\section{Tolerantly Testing Isomorphism to Functions with Small Spectral Norm}

In order to talk about tolerantly testing $g$-isomorphism, say that $f$ is $(\tau, g)$-isomorphic if $f$ is $\tau$-close to a $g$-isomorphic function. In this section, we show the following tolerant tester for $g$-isomorphism.

Theorem 5. Let $g: \mathbb{F}_{2}^{n} \rightarrow\{-1,1\}$ be a function with $\widehat{\|}_{\mathrm{N}} \widehat{\|}_{1} \leq s$. Then, there is an algorithm with query complexity $\widetilde{O}\left((s / \epsilon)^{24}\right)$ such that, given a function $f$, it accepts with probability at least $\frac{2}{3}$ when $f$ is $\left(\frac{\epsilon}{3}, g\right)$-isomorphic and rejects with probability at least $\frac{2}{3}$ when $f$ is not $\left(\frac{2 \epsilon}{3}, g\right)$-isomorphic.

Our algorithm is given in Algorithm 2. We start by running Algorithm 1 with $\theta=\frac{\epsilon}{6 s}$ and $m=\widetilde{O}\left(\frac{s^{2}}{\epsilon^{2}}\right)$, and then we run an extra step: we use the examples to estimate each heavy Fourier coefficient to within $\frac{\epsilon}{3 s}$. We estimate every other Fourier coefficient to be 0 . Because we $\operatorname{set} \theta=\frac{\epsilon}{6 s}$, 
all estimates are correct to within $\frac{\epsilon}{3 s}$, (except with probability at most $\frac{1}{100}$, if we draw enough examples) because the small Fourier coefficients estimated to be 0 without any examples have magnitude at most $\frac{\epsilon}{6 s}$. The probability that any of the $\left|\mathcal{L}^{\prime}\right|$ estimates is outside the stated interval is at most $\frac{1}{100}$.

We will assume that we have $g$ given as a multilinear polynomial; $g=\sum_{\beta \in \mathbb{F}_{2}^{n}} \widehat{g}(\beta) \chi_{\beta}$. The algorithm tries to find the best match of the estimates of the Fourier coefficients found to the Fourier coefficients of $g$. An issue here is that we do not actually know $\alpha(C)$ 's and hence it is not immediate how to execute Step 5. A key insight is that, to discuss linear transformations applied to these heavy coefficients, it suffices to determine the linear relationships between the coefficients. Proofs of the following lemmas are deferred to Appendix C.

Lemma 9. Let $B=\left\{C_{1}, C_{2}, \ldots, C_{k}\right\} \subseteq \mathcal{L}^{\prime}$ be a minimal collection of buckets such that $\alpha\left(\mathcal{L}^{\prime}\right) \subseteq \operatorname{span}\left(\left\{\alpha\left(C_{1}\right), \alpha\left(C_{2}\right), \ldots, \alpha\left(C_{k}\right)\right\}\right)$.

Suppose that $\mathcal{M}$ is chosen uniformly at random in Implicit Sieve. Then the rows of $\mathcal{Q}$ are uniformly distributed when restricted to the columns in $B$, and every other entry in a fixed row is determined by the setting of the bits in the columns in $B$.

Lemma 10. Let $\mathcal{S}=\left\{S \mid S \subseteq \alpha\left(\mathcal{L}^{\prime}\right)\right.$ and $\left.\sum_{\alpha \in S} \alpha=0^{n}\right\}$, where the sum is in the $\mathbb{F}_{2}^{n}$ sense. Except with probability at most $\frac{1}{100}$ over the construction of $\mathcal{Q}$, we can find sets of buckets corresponding to $\mathcal{S}$ from $\mathcal{Q}$.

Lemma 11. The algorithm is able to perform Step 5 by enumerating over all linear transformations without using any further queries.

The following lemma shows that if $f$ is close to $g$, then there is a very good match between the Fourier coefficients of $f$ and $g$.

Lemma 12. Let $g: \mathbb{F}_{2}^{n} \rightarrow\{-1,1\}$ be a fixed function, and suppose $f$ is $\left(\frac{\epsilon}{3}, g\right)$-isomorphic; further, let $A$ be a linear transformation such that $f \circ A$ is $\frac{\epsilon}{3}$-close to $g$. Then $\sum_{\alpha \in \operatorname{supp}(g)} \widehat{f \circ A}(\alpha) \widehat{g}(\alpha) \geq 1-\frac{2 \epsilon}{3}$.

Proof. Because $f \circ A$ and $g$ are $\{+1,-1\}$-valued, $\operatorname{Pr}_{\boldsymbol{x}}[(f \circ A)(\boldsymbol{x}) \neq g(\boldsymbol{x})]=$ $\frac{1}{2}+\frac{1}{2} \mathbf{E}_{\boldsymbol{x}}[(f \circ A)(\boldsymbol{x}) g(\boldsymbol{x})]$. It follows that if $(f \circ A)$ and $g$ are $\frac{\epsilon}{3}$-close, then $\mathbf{E}_{\boldsymbol{x}}[(f \circ A)(\boldsymbol{x}) g(\boldsymbol{x})] \geq 1-\frac{2 \epsilon}{3}$. But $\mathbf{E}_{\boldsymbol{x}}[(f \circ A)(\boldsymbol{x}) g(\boldsymbol{x})]=\sum_{\alpha \in \mathbb{F}_{2}^{n}} \widehat{f \circ A}(\alpha) \widehat{g}(\alpha)$, finishing the proof.

Theorem 5 follows from the following two lemmas. 
Lemma 13. Suppose that $f$ is $\left(\frac{\epsilon}{3}, g\right)$-isomorphic, where $\widehat{\|}_{\|} \widehat{\|}_{1} \leq s$. Then the algorithm accepts with probability at least $\frac{2}{3}$.

Proof. We assume that $(\mathcal{Q}, \mathcal{F})$ is found correctly, that estimates in Algorithm 2 are correct, and that all linear dependencies are discovered. The probability of failing at least one of these is at most $\frac{6}{100}+\frac{1}{100}+\frac{1}{100}=\frac{8}{100}$.

Because $f$ is $\left(\frac{\epsilon}{3}, g\right)$-isomorphic, there is a nonsingular linear transformation $A$ such that $f \circ A$ is $\frac{\epsilon}{3}$-close to $g$. By Lemma $12, \sum_{\beta \in \operatorname{supp}(g)} \widehat{f \circ A}(\beta) \widehat{g}(\beta) \geq$ $1-\frac{2 \epsilon}{3}$.

We only have estimates for each $\widehat{f \circ A}(\beta)$, and each could be off by at most $\frac{\epsilon}{3 s}$. Because $\widehat{\|} g \widehat{\|}_{1} \leq s$, using these estimates can reduce this "dot

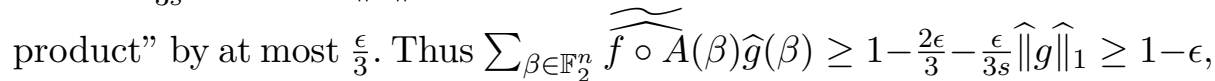
so the algorithm will accept. Thus, the algorithm accepts with probability at least $1-\frac{8}{100}>\frac{2}{3}$.

Lemma 14. Suppose that the algorithm accepts with probability at least $\frac{1}{3}$. Then $f$ is $\left(\frac{2 \epsilon}{3}, g\right)$-isomorphic.

Proof. Again, we assume that $(\mathcal{Q}, \mathcal{F})$ is found correctly, that the estimates in Algorithm 2 are correct, and that all linear dependencies are discovered. The probability of failing at least one of these is at most $\frac{6}{100}+\frac{1}{100}+\frac{1}{100}=\frac{8}{100}$. Suppose the algorithm accepts with probability at least $\frac{1}{3}>\frac{8}{100}$. Then, with nonzero probability, the algorithm finds a

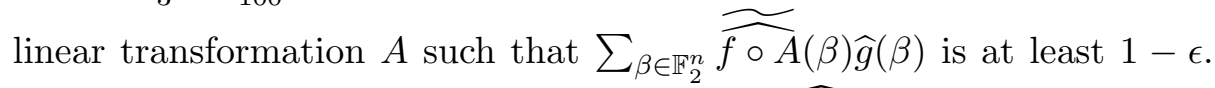
As in the previous claim, each estimate of $\widehat{f \circ A}$ is within $\frac{\epsilon}{3 s}$ of the true value, so $\sum_{\beta \in \mathbb{F}_{2}^{n}} \widehat{f \circ A}(\beta) \widehat{g}(\beta) \geq 1-\epsilon-\frac{\epsilon}{3 s} \widehat{\|}_{g} \widehat{\|}_{1} \geq 1-\frac{4 \epsilon}{3}$. Then, $\sum_{\beta \in \mathbb{F}_{2}^{n}}(\widehat{f \circ A}(\beta)-\widehat{g}(\beta))^{2}=\sum_{\beta \in \mathbb{F}_{2}^{n}} \widehat{f \circ A}(\beta)^{2}-2 \sum_{\beta \in \mathbb{F}_{2}^{n}} \widehat{f \circ A}(\beta) \widehat{g}(\beta)+$ $\sum_{\beta \in \mathbb{F}_{2}^{n}} \widehat{g}(\beta)^{2} \leq 2-2\left(1-\frac{4 \epsilon}{3}\right)=\frac{8 \epsilon}{3}$. It follows that $\operatorname{Pr}_{\boldsymbol{x}}[(f \circ A)(\boldsymbol{x}) \neq g(\boldsymbol{x})]=$ $\frac{1}{4} \mathbf{E}_{\boldsymbol{x}}\left[((f \circ A)(\boldsymbol{x})-g(\boldsymbol{x}))^{2}\right]=\frac{1}{4} \sum_{\beta \in \mathbb{F}_{2}^{n}}(\widehat{f \circ A}(\beta)-\widehat{g}(\beta))^{2} \leq \frac{1}{4} \cdot \frac{8 \epsilon}{3}=\frac{2 \epsilon}{3}$, and thus $f$ is $\left(\frac{2 \epsilon}{3}, g\right)$-isomorphic.

Proof (of Theorem 1). Suppose that $g$ is $\frac{\epsilon}{3}$-close to a function $h$ with $\widehat{\|} h \widehat{\|}_{1} \leq s$. To test whether the input function $f$ is isomorphic to $g$, we instead test $\left(\frac{\epsilon}{3}, h\right)$-isomorphism using Theorem 5. If $f$ is isomorphic to $g$, then $f$ is $\frac{\epsilon}{3}$-close to $h$, and we accept with probability at least $\frac{2}{3}$ from Lemma 13. Suppose that $f$ is $\epsilon$-far from being isomorphic $g$. Then, $f$ is $\frac{2 \epsilon}{3}$-far from being isomorphic to $h$ from the triangle inequality. Thus, we reject with probability at least $\frac{2}{3}$ from Lemma 14 . 


\section{References}

1. Rubinfeld, R., Sudan, M.: Robust characterizations of polynomials with applications to program testing. SIAM J. Comput. 25(2) (1996) 252-271

2. Goldreich, O., Goldwasser, S., Ron, D.: Property testing and its connection to learning and approximation. J. ACM 45(4) (1998) 653-750

3. Ron, D.: Algorithmic and analysis techniques in property testing. Foundations and Trends in Theoretical Computer Science 5 (2010) 73-205

4. Rubinfeld, R., Shapira, A.: Sublinear time algorithms. Electronic Colloquium on Computational Complexity (ECCC) 18 (2011) TR11-013.

5. Goldreich, O., ed.: Property Testing: Current Research and Surveys. Volume 6390 of LNCS. Springer (2010)

6. Alon, N., Fischer, E., Newman, I., Shapira, A.: A combinatorial characterization of the testable graph properties: It's all about regularity. SIAM J. Comput. 39 (2009) 143-167

7. Bhattacharyya, A., Grigorescu, E., Shapira, A.: A unified framework for testing linear-invariant properties. In: FOCS. (2010) 478-487

8. Grigorescu, E., Kaufman, T., Sudan, M.: 2-transitivity is insufficient for local testability. In: CCC. (2008) 259-267

9. Kaufman, T., Sudan, M.: Algebraic property testing: the role of invariance. In: STOC. (2008) 403-412

10. Sudan, M.: Invariance in property testing. Property testing (2011) 211-227

11. Fischer, E.: The difficulty of testing for isomorphism against a graph that is given in advance. In: STOC. (2004) 391-397

12. Fischer, E., Newman, I.: Testing versus estimation of graph properties. SIAM J. Comput. 37(2) (2008) 482-501

13. Gopalan, P., O’Donnell, R., Servedio, R., Shpilka, A., Wimmer, K.: Testing fourier dimensionality and sparsity. SIAM J. Comput. 40(4) (2011) 1075-1100

14. Diakonikolas, I., Lee, H., Matulef, K., Onak, K., Rubinfeld, R., Servedio, R., Wan, A.: Testing for concise representations. In: FOCS. (2007) 549-558

15. Goldreich, O., Levin, L.: A hard-core predicate for all one-way functions. In: STOC. (1989) 25-32

16. Kushilevitz, E., Mansour, Y.: Learning decision trees using the fourier spectrum. In: STOC. (1991) 455-464

17. Fischer, E., Kindler, G., Ron, D., Safra, S., Samorodnitsky, A.: Testing juntas. Journal of Computer and System Sciences 68(4) (2004) 753-787

18. Blais, E., Weinstein, A., Yoshida, Y.: Partially symmetric functions are efficiently isomorphism-testable. In: FOCS. (2012) 551-560.

19. Chakraborty, S., Fischer, E., Garća-Soriano, D., Matsliah, A.: Junto-symmetric functions, hypergraph isomorphism, and crunching. In: CCC. (2012) 148-158

20. Grigorescu, E., Wimmer, K., Xie, N.: Tight lower bounds for testing linear isomorphism Manuscript.

21. Bhattacharyya, A., Fischer, E., Hatami, H., Hatami, P., Lovett, S.: Every locally characterized affine-invariant property is testable. In: STOC. (2013) to appear.

22. Fischer, E., Matsliah, A.: Testing graph isomorphism. In: SODA. (2006) 299-308

23. Newman, I., Sohler, C.: Every property of hyperfinite graphs is testable. In: STOC. (2011) 675-684 


\section{A Proofs from Preliminaries}

See Fact 2.3 of [13] for the following fact.

Claim. $\mathrm{P}_{r+H} f(x)=\mathbf{E}_{\boldsymbol{y} \in H^{\perp}}\left[\chi_{r}(\boldsymbol{y}) f(x+\boldsymbol{y})\right]$.

Proof (of Lemma 1).

$$
\begin{aligned}
\widehat{f \circ A}(\alpha) & =\underset{\boldsymbol{x}}{\mathbf{E}}\left[f(A x) \chi_{\alpha}(\boldsymbol{x})\right]=\underset{\boldsymbol{x}}{\mathbf{E}}\left[f(\boldsymbol{x}) \chi_{\alpha}\left(A^{-1} \boldsymbol{x}\right)\right]=\underset{\boldsymbol{x}}{\mathbf{E}}\left[f(\boldsymbol{x})(-1)^{\left\langle\alpha, A^{-1} \boldsymbol{x}\right\rangle}\right] \\
& =\underset{\boldsymbol{x}}{\mathbf{E}}\left[f(\boldsymbol{x})(-1)^{\left\langle\left(A^{-1}\right)^{T} \alpha, \boldsymbol{x}\right\rangle}\right]=\underset{\boldsymbol{x}}{\mathbf{E}}\left[f(\boldsymbol{x}) \chi_{\left(A^{-1}\right)^{T} \alpha}(\boldsymbol{x})\right]=\widehat{f}\left(\left(A^{-1}\right)^{T} \alpha\right)
\end{aligned}
$$

The second claim is obvious.

Proof (of Lemma 2). We note that

$$
\begin{aligned}
\mathrm{wt}_{2}(r+H) & =\sum_{\alpha \in r+H} \widehat{f}(\alpha)^{2}=\underset{\boldsymbol{w} \in \mathbb{F}_{2}^{n}}{\mathbf{E}}\left[P_{r+H} f(\boldsymbol{w})^{2}\right] \\
& =\underset{\boldsymbol{w} \in \mathbb{F}_{2}^{n}, \boldsymbol{y}_{1}, \boldsymbol{y}_{2} \in H^{\perp}}{\mathbf{E}}\left[\chi_{r}\left(\boldsymbol{y}_{1}\right) f\left(\boldsymbol{w}+\boldsymbol{y}_{1}\right) \chi_{r}\left(\boldsymbol{y}_{2}\right) f\left(\boldsymbol{w}+\boldsymbol{y}_{2}\right)\right]
\end{aligned}
$$

by Parseval, and

$$
\begin{aligned}
& \operatorname{wt}_{4}(r+H)=\sum_{\alpha \in r+H} \widehat{f}(\alpha)^{4} \\
&=\underset{\boldsymbol{w}_{1}, \boldsymbol{w}_{2}, \boldsymbol{w}_{3} \in \mathbb{F}_{2}^{n}}{\mathbf{E}}\left[P_{r+H} f\left(\boldsymbol{w}_{1}\right) P_{r+H} f\left(\boldsymbol{w}_{2}\right) P_{r+H} f\left(\boldsymbol{w}_{3}\right) P_{r+H} f\left(\boldsymbol{w}_{1}+\boldsymbol{w}_{2}+\boldsymbol{w}_{3}\right)\right] \\
&=\underset{\substack{\boldsymbol{w}_{1}, \boldsymbol{w}_{2}, \boldsymbol{w}_{3} \in \mathbb{F}_{2}^{n}, \boldsymbol{y}_{1}, \boldsymbol{y}_{2}, \boldsymbol{y}_{3}, \boldsymbol{y}_{4} \in H^{\perp}}}{\mathbf{E}}\left[\chi_{r}\left(\boldsymbol{y}_{1}\right) f\left(\boldsymbol{w}_{1}+\boldsymbol{y}_{1}\right) \chi_{r}\left(\boldsymbol{y}_{2}\right) f\left(\boldsymbol{w}_{2}+\boldsymbol{y}_{2}\right) \chi_{r}\left(\boldsymbol{y}_{3}\right)\right. \\
&\left.\cdot f\left(\boldsymbol{w}_{3}+\boldsymbol{y}_{3}\right) \chi_{r}\left(\boldsymbol{y}_{4}\right) f\left(\boldsymbol{w}_{1}+\boldsymbol{w}_{2}+\boldsymbol{w}_{3}+\boldsymbol{y}_{4}\right)\right]
\end{aligned}
$$

The quantities inside each expectation are bounded in $[-1,1]$, so the result follows from an application of a Chernoff bound.

\section{B Proofs from Section 3}

We use the following fact to show Lemma 4.

Lemma 15 ([13]). Let $(H, \mathcal{C})$ be a random permuted $t$-dimensional coset structure, where $t \geq 2 \log s+\log \frac{1}{\delta}$. Then for any set $S \subseteq \mathbb{F}_{2}^{n}$ where $|S| \leq s+1$, every vector in $S$ gets mapped into a different bucket with probability at least $1-\delta$. 
Proof (of Lemma 4). By Parseval, $\sum_{\alpha \in \mathbb{F}_{2}^{n}} \widehat{f}(\alpha)^{2}=1$, so for the set $S$ as defined above, $|S| \leq \frac{16}{\theta^{4}}$. The claim now follows by Lemma 15 .

Proof (of Lemma 5). We say that a Fourier coefficient $\widehat{f}(\alpha)$ is big if $|\widehat{f}(\alpha)| \geq \frac{1}{4} \theta^{2}$ and small otherwise. There are at most $\frac{16}{\theta^{4}}$ big coefficients regardless of $t$. By assumption, $t$ is large enough so that by Lemma 4, the big Fourier coefficients are all in different buckets with high probability. We will assume that this event happens. Using Chebyshev's inequality, we can show that the small Fourier coefficients are close to uniformly distributed for the heavy buckets.

Suppose that a Fourier coefficient $\widehat{f}(\alpha)$ is big for some $\alpha \in \mathbb{F}_{2}^{n}$ and let $C_{\alpha}$ be the bucket $\alpha$ belongs to. Let $b \in \mathbb{F}_{2}^{t}$ be the vector corresponding to $C_{\alpha}$. That is, $\left\langle\alpha, \beta_{i}\right\rangle=b_{i}$ for every $i \in\{1, \ldots, t\}$, where $\beta_{i}$ 's are vectors used to define a random $t$-dimensional coset structure. Note that $C_{\alpha}$ is a random variable. Define $S=\left\{\gamma \in \mathbb{F}_{2}^{n}|| \widehat{f}(\gamma) \mid<\frac{\theta^{2}}{4}\right\}$. For every $\gamma \in S$ and every bucket $D$, let $X_{\gamma, D}$ be the random variable that is $\widehat{f}(\gamma)^{2}$ if $\gamma$ is in $D$ and 0 otherwise.

Let $X_{C_{\alpha}}=\sum_{\gamma \in S} X_{\gamma, C_{\alpha}}$. Now, we want to bound the probability that $X_{C_{\alpha}}$ deviates much from its expectation.

Let $\mathbf{0}$ denote the zero vector, and let $C_{0}$ be the bucket corresponding to the zero vector $\mathbf{0}$.

We first assume that $\widehat{f}(\mathbf{0})$ is not big, so $\alpha \neq \mathbf{0}$. For $\alpha \neq \mathbf{0}$, we will simply treat the case that $C_{\alpha}=C_{0}$ as a bad event; this occurs with probability $2^{-t}$ when $\alpha \neq \mathbf{0}$ (as we have assumed). We will handle $C_{0}$ as a special case, as well as the case where $\widehat{f}(\mathbf{0})$ is big.

Now, we bound $\operatorname{Var}\left[X_{C_{\alpha}} \mid C_{\alpha} \neq C_{0}\right]$. To this end, we consider when $X_{\gamma_{1}, C_{\alpha}}$ and $X_{\gamma_{2}, C_{\alpha}}$ are independent conditioned on $C_{\alpha} \neq C_{0}$, where $\gamma_{1} \neq$ $\gamma_{2} \in \mathbb{F}_{2}^{n}$. Suppose that $\gamma_{2}=\gamma_{1}+\alpha$. In this case, since $\left\langle\gamma_{2}, \beta_{i}\right\rangle=\left\langle\gamma_{1}+\right.$ $\left.\alpha, \beta_{i}\right\rangle=\left\langle\gamma_{1}, \beta_{i}\right\rangle+\left\langle\alpha, \beta_{i}\right\rangle$, the bucket $\gamma_{2}$ belongs to depends on the buckets $\gamma_{1}$ and $\alpha$ belong to. Thus, $X_{\gamma_{1}, C_{\alpha}}$ and $X_{\gamma_{2}, C_{\alpha}}$ are not independent. On the other hand, if $\gamma_{2} \neq \gamma_{1}+\alpha$, the bucket $\gamma_{2}$ belongs to is independent of the bucket $\gamma_{1}$ belongs to. Thus, $X_{\gamma_{1}, C_{\alpha}}$ and $X_{\gamma_{2}, C_{\alpha}}$ are independent. To see this, let $\gamma_{1} \neq \gamma_{2}$ be such that $\gamma_{2} \neq \gamma_{1}+\alpha$. It follows that $\gamma_{1}+\gamma_{2}+\alpha \neq$ $\mathbf{0}$, and $X_{\gamma_{1}, C_{\alpha}}$ and $X_{\gamma_{2}, C_{\alpha}}$, are independent if $\left\langle\gamma_{1}+\gamma_{2}, \beta_{i}\right\rangle$ is uniformly random, conditioned on the distribution of $\beta_{i}$ being uniformly random given $\left\langle\alpha, \beta_{i}\right\rangle=b_{i}$. This is true; $\left\langle\gamma_{1}+\gamma_{2}, \beta_{i}\right\rangle=\left\langle\gamma_{1}+\gamma_{2}+\alpha, \beta_{i}\right\rangle+\left\langle\alpha, \beta_{i}\right\rangle$, and if $\gamma_{2} \neq \gamma_{1}+\alpha$ (and we assume $\gamma_{1} \neq \gamma_{2}$ ), the first inner product has the uniform distribution. As a corollary, for the fixed bucket $C_{0}, X_{\gamma_{1}, C_{0}}$ and $X_{\gamma_{2}, C_{0}}$ are independent if $\gamma_{1} \neq \gamma_{2}$. 
Therefore,

$$
\begin{aligned}
& \operatorname{Var}\left[X_{C_{\alpha}} \mid C_{\alpha} \neq C_{0}\right]=\operatorname{Var}\left[\sum_{\gamma \in S} X_{\gamma, C_{\alpha}} \mid C_{\alpha} \neq C_{0}\right] \\
= & \mathbf{E}\left[\left(\sum_{\gamma \in S} X_{\gamma, C_{\alpha}}\right)^{2} \mid C_{\alpha} \neq C_{0}\right]-\mathbf{E}\left[\sum_{\gamma \in S} X_{\gamma, C_{\alpha}} \mid C_{\alpha} \neq C_{0}\right]^{2} \\
= & \sum_{\gamma_{1}, \gamma_{2} \in S} \mathbf{E}\left[X_{\gamma_{1}, C_{\alpha}} X_{\gamma_{2}, C_{\alpha}} \mid C_{\alpha} \neq C_{0}\right]- \\
& \sum_{\gamma_{1}, \gamma_{2} \in S} \mathbf{E}\left[X_{\gamma_{1}, C_{\alpha}} \mid C_{\alpha} \neq C_{0}\right] \mathbf{E}\left[X_{\gamma_{2}, C_{\alpha}} \mid C_{\alpha} \neq C_{0}\right] \\
\leq & \sum_{\gamma \in S} \mathbf{E}\left[X_{\gamma, C_{\alpha}}^{2} \mid C_{\alpha} \neq C_{0}\right]+\sum_{\gamma \in S} \mathbf{E}\left[X_{\gamma, C_{\alpha}} X_{\gamma+\alpha, C_{\alpha}} \mid C_{\alpha} \neq C_{0}\right] .
\end{aligned}
$$

For the first term, since $\left|X_{\gamma, C_{\alpha}}\right| \leq \frac{1}{16} \theta^{4}$ for $\gamma \in S$ and $\mathbf{E}\left[X_{C_{\alpha}} \mid C_{\alpha} \neq\right.$ $\left.C_{0}\right]=\sum_{\gamma \in S} \mathbf{E}\left[X_{\gamma, C_{\alpha}} \mid C_{\alpha} \neq C_{0}\right] \leq 2^{-t}$, we have

$$
\sum_{\gamma \in S} \mathbf{E}\left[X_{\gamma, C_{\alpha}}^{2} \mid C_{\alpha} \neq C_{0}\right] \leq \sum_{\gamma \in S} \frac{1}{16} \theta^{4} \mathbf{E}\left[X_{\gamma, C_{\alpha}} \mid C_{\alpha} \neq C_{0}\right] \leq \frac{1}{16} \theta^{4} 2^{-t}
$$

Now consider the second term. $X_{\gamma, C_{\alpha}} X_{\gamma+\alpha, C_{\alpha}}$ can be non-zero only when $\gamma \in C_{\alpha}$ and $\gamma+\alpha \in C_{\alpha}$. If $\gamma \in C_{\alpha}$, then $\left\langle\gamma, \beta_{i}\right\rangle=b_{i}$ for every $i \in\{1, \ldots, t\}$. Thus, $\left\langle\gamma+\alpha, \beta_{i}\right\rangle=2 b_{i}=0(\bmod 2)$. Then, $\gamma$ and $\gamma+\alpha$ cannot be in the same bucket when $C_{\alpha} \neq C_{0}$. and thus the second term is zero.

Using Chebyshev's inequality with $d=\frac{40}{\theta^{2}}$, we have

$$
\begin{aligned}
& \operatorname{Pr}\left[X_{C_{\alpha}}>10 \cdot 2^{-t / 2}+2^{-t} \mid C_{\alpha} \neq C_{0}\right] \\
\leq & \operatorname{Pr}\left[X_{C_{\alpha}}-\mathbf{E}\left[X_{C_{\alpha}}\right]>d \sqrt{\operatorname{Var}[X]} \mid C_{\alpha} \neq C_{0}\right] \\
\leq & \frac{1}{d^{2}}=\frac{\theta^{4}}{1600} .
\end{aligned}
$$

Here, we use the fact that $\mathbf{E}\left[X_{C_{\alpha}} \mid C_{\alpha} \neq C_{0}\right] \leq 2^{-t}$.

We choose $t$ large enough so that $10 \cdot 2^{-t / 2}+2^{-t} \leq\left(\frac{\theta^{3}}{3200 m}\right)^{2}$. Since the probability that $C_{\alpha}=C_{0}$ is exactly $2^{-t}$, for any fixed $\alpha \neq \mathbf{0}, \operatorname{wt}_{2}\left(C_{\alpha}\right) \leq$ $\widehat{f}\left(\alpha\left(C_{\alpha}\right)\right)^{2}+\left(\frac{\theta^{3}}{3200 m}\right)^{2}$ except with probability $\frac{\theta^{4}}{1600}+2^{-t} \leq \frac{\theta^{4}}{800}$. Union bounding over the $\frac{16}{\theta^{4}}$ big coefficients shows that the claim is true for all heavy buckets except with probability $\frac{2}{100}$.

In the case that $\widehat{f}(\mathbf{0})$ is big, then for every other big $\alpha$, we have $C_{\alpha} \neq C_{0}$ with certainty, since we assumed that the big Fourier coefficients 
are in different buckets. The above analysis works for $C_{0}$ by applying the same analysis as above, removing the $C_{\alpha} \neq C_{0}$ condition and replacing $X_{C_{\alpha}}$ and $X_{\gamma, C_{\alpha}}$ with $X_{C_{0}}$ and $X_{\gamma, C_{0}}$ respectively in (1), (2), and (3). In fact, this analysis is wasteful; Since the $X_{\gamma, C_{0}}$ variables are pairwise independent, the third line of the expression(s) in (1) can be bounded by $\sum_{\gamma \in S} \mathbf{E}\left[X_{\gamma, C_{0}}^{2}\right]$, which is at most $\frac{1}{16} \theta^{4} 2^{-t}$.

Proof (of Lemma 6). We first show the first part of the lemma. Note that because $\widetilde{\mathrm{wt}}_{2}(C) \geq \frac{3}{4} \theta^{2}$ and this estimate is accurate within $\frac{\theta^{2}}{4}$, we have $\mathrm{wt}_{2}(C) \leq 2 \widetilde{\mathrm{wt}}_{2}(C)$. Thus,

$$
\begin{aligned}
& \widetilde{\mathrm{wt}}_{4}(C) \leq \mathrm{wt}_{4}(C)+\frac{1}{4} \widetilde{\mathrm{wt}}_{2}(C) \theta^{4} \leq \mathrm{wt}_{2}(C) \underset{\alpha \in C}{\max } \widehat{f}(\alpha)^{2}+\frac{1}{4} \widetilde{\mathrm{wt}}_{2}(C) \theta^{4} \\
\leq & \frac{1}{16} \mathrm{wt}_{2}(C) \theta^{4}+\frac{1}{4} \widetilde{\mathrm{wt}}_{2}(C) \theta^{4} \leq \frac{1}{8} \widetilde{\mathrm{wt}}_{2}(C) \theta^{4}+\frac{1}{4} \widetilde{\mathrm{wt}_{2}}(C) \theta^{4}=\frac{3}{8} \widetilde{\mathrm{wt}}_{2}(C) \theta^{4} \\
\leq & \frac{3}{8} \theta^{4},
\end{aligned}
$$

and Find-Heavy-Buckets will discard $C$. We make at most $2 \cdot 2^{t}$ estimates with failure probability $\frac{1}{200 \cdot 2^{t}}$, so the total failure probability in Steps 2 through 7 is at most $\frac{1}{100}$.

If $C$ contains a vector $\alpha$ such that $|\widehat{f}(\alpha(C))| \geq \theta$, then

$$
\widetilde{\mathrm{wt}}_{4}(C) \geq \mathrm{wt}_{4}(C)-\frac{1}{4} \widetilde{\mathrm{wt}}_{2}(C) \theta^{4} \geq \theta^{4}-\frac{1}{4} \widetilde{\mathrm{wt}}_{2}(C) \theta^{4} \geq \frac{3}{4} \theta^{4},
$$

giving the second part of the lemma.

We now show the third part of the lemma. Let $C$ be a bucket such that $|\widehat{f}(\alpha(C))|<\frac{1}{2} \theta$. By Lemma $5, \mathrm{wt}_{2}(C) \leq \widehat{f}(\alpha(C))^{2}+\left(\frac{\theta^{3}}{3200 m}\right)^{2}<$ $\frac{1}{4} \theta^{2}+\frac{1}{4} \theta^{2}=\frac{1}{2} \theta^{2}$. Thus, in Step $3, \widetilde{\mathrm{wt}}_{2}(C) \leq \mathrm{wt}_{2}(C)+\frac{1}{4} \theta^{2}<\frac{3}{4} \theta^{2}$, so $C$ is discarded in Step 4.

Proof (of Lemma 7). Note that $\mathbf{E}\left[S_{C}(f)^{2}\right] \leq\left(\frac{\theta^{3}}{3200 m}\right)^{2}$ by Lemma 5 . By Cauchy-Schwarz, $\mathbf{E}\left[\left|S_{C}(f)\right|\right] \leq \frac{\theta^{3}}{3200 m}$, and by Markov's inequality, $\operatorname{Pr}\left[\left|S_{C}(f)(\boldsymbol{x})\right| \geq \frac{1}{4} \theta\right] \leq \frac{\theta^{2}}{800 \mathrm{~m}}$ as claimed.

Proof (of Lemma 8). Assume the condition inside the probability mentioned in Lemma 7 does not hold; that is, assume $S_{C}(f)(x)<\frac{1}{4} \theta$. For a surviving bucket $C$, we have that $\mathrm{P}_{C}(f)(x)=\widehat{f}(\alpha(C)) \chi_{\alpha(C)}(x)+S_{C}(f)(x)$ and $|\widehat{f}(\alpha(C))| \geq \frac{1}{2} \theta$. It follows that $\left|\mathrm{P}_{C}(f)(x)\right| \geq|\widehat{f}(\alpha(C))|-\left|S_{C}(f)(x)\right| \geq$ $\frac{1}{2} \theta-\frac{1}{4} \theta=\frac{1}{4} \theta$. Also, $|\widehat{f}(\alpha(C))| \geq\left|S_{C}(f)(x)\right|, \operatorname{so~} \operatorname{sgn}\left(\mathrm{P}_{C}(f)(x)\right)=\operatorname{sgn}\left(\widehat{f}(\alpha(C)) \chi_{\alpha(C)}(x)\right)$. Thus the probability in the claim is at most $\frac{\theta^{2}}{800 m}$ from Lemma 7 . 


\section{Proofs from Section 4}

Proof (of Lemma 9). We show the first part. Since $\alpha\left(C_{i}\right), \ldots, \alpha\left(C_{k}\right)$ are linearly independent, the distribution of the tuple $\left(\chi_{\alpha\left(C_{1}\right)}(x), \ldots, \chi_{\alpha\left(C_{k}\right)}(x)\right)$ is uniform when $x$ is uniformly distributed.

For the second, we use the fact that $\left.\operatorname{span}\left(\left\{\alpha\left(C_{1}\right), \alpha\left(C_{2}\right), \ldots, \alpha\left(C_{k}\right)\right\}\right)\right)$ contains $\alpha\left(\mathcal{L}^{\prime}\right)$. Each row of $\mathcal{Q}$ corresponds to evaluation of a linear function on some string $x_{i} \in \mathbb{F}_{2}^{n}$, and each column corresponds to a linear (in an $\mathbb{F}_{2}$ sense) function. Thus every other entry can be inferred from the entries in columns corresponding to $B$ using linearity.

Proof (of Lemma 10). For $S \subseteq \alpha\left(\mathcal{L}^{\prime}\right)$, define $\alpha_{S}:=\sum_{\alpha \in S} \alpha$. Note that for $\alpha_{S}=0^{n}$ if and only if $S \in \mathcal{S}$. We claim that, except with probability $\frac{1}{100}, \mathcal{S}$ is simply every subset $S \subseteq \alpha\left(\mathcal{L}^{\prime}\right)$ such that there is no $x_{i}$ where $\prod_{\alpha \in S} \chi_{\alpha}\left(x_{i}\right)=-1$. (Here, we take the product as real numbers as an analogue of addition in $\mathbb{F}_{2}$, where we identify $-1 \in \mathbb{R}$ with $1 \in \mathbb{F}_{2}$ and $1 \in \mathbb{R}$ with $0 \in \mathbb{F}_{2}$.) This is clearly true with certainty for every $S \in \mathcal{S}$.

If $S \notin \mathcal{S}$, then when $\boldsymbol{x}_{i}$ is drawn uniformly at random, $\prod_{\alpha \in S} \chi_{\alpha}\left(\boldsymbol{x}_{i}\right)$ is equally likely to be -1 and 1 by the previous lemma. Thus, the probability that -1 never shows up as the product of the entries of a fixed row is $2^{-m}$, where $m=\widetilde{O}\left(\frac{s^{2}}{\epsilon^{2}}\right)$. There are at most $2^{O\left(s^{2} / \epsilon^{2}\right)}$ subsets of columns of $\mathcal{Q}$, since each column corresponds to a Fourier coefficient of magnitude at least $\frac{\epsilon}{6 s}$ (and applying Parseval). So by taking the hidden constant in $m$ large enough, by the union bound the probability that a linearly independent set is missed is at most $\frac{1}{100}$.

Algorithmically, we can simply exhaustively consider every subset of $\mathcal{L}^{\prime}$ for linear dependence, which will yield the linear structure of $\alpha\left(\mathcal{L}^{\prime}\right)$.

Proof (of Lemma 11). While we have implicit access to $f$, we can consider every nonsingular linear transformation applied to the vectors of $\alpha\left(\mathcal{L}^{\prime}\right)$ (although the actual transformation applied is still implicit.). By Lemma 9 , there is a minimal collection of buckets $B=\left\{C_{1}, C_{2}, \ldots, C_{k}\right\}$ such that $\alpha\left(\mathcal{L}^{\prime}\right) \subseteq \operatorname{span}(B)$. By Lemma 10, we can find such a set $B$, except with probability at most $\frac{1}{100}$. We can take $B$ to be an arbitrary maximal subset of $\alpha\left(\mathcal{L}^{\prime}\right)$ with no linear dependencies; we find all the linear dependencies when we find $\mathcal{S}$ as in Lemma 10. Once we have such a set $B$, we consider all linear transformations formed by choosing the images of $\left\{\alpha\left(C_{1}\right), \alpha\left(C_{2}\right), \ldots, \alpha\left(C_{k}\right)\right\}$ and applying linearity. We will denote such linear transformation by $A$; we stress that the algorithm does not know the specific form of $A$ since we do not know what $\alpha\left(C_{i}\right), \ldots, \alpha\left(C_{k}\right)$ are. In fact, the other $n-k$ dimensions are irrelevant; the algorithm needs 
$\overline{\text { Algorithm } 3 \text { Tolerant } g \text {-isomorphism testing to a function with small }}$ spectral norm for multiple $g$

Parameters: $s, \mathcal{G} \subseteq\left\{g: \mathbb{F}_{2}^{n} \rightarrow\{-1,1\} \mid \widehat{\|} g \widehat{\|}_{1} \leq s\right\}, \epsilon$

1: Run Algorithm 1 with $\theta=\frac{\epsilon}{6 s}, m=\widetilde{O}\left(\frac{s^{2}}{\epsilon^{2}}\right)$, and $\mathcal{M}$ a uniformly random subset of $\mathbb{F}_{2}^{n}$ with $|\mathcal{M}|=m$ to get $(\mathcal{Q}, \mathcal{F})$.

2: for each $C \in \mathcal{L}^{\prime}$ do

3: $\quad$ Use $(\mathcal{Q}, \mathcal{F})$ to estimate $\widehat{f}(\alpha(C))$ within $\pm \frac{\epsilon}{3 s}$ with confidence $1-\frac{1}{100\left|\mathcal{L}^{\prime}\right|}$ and call it $\widetilde{\widehat{f}}(\alpha(C))$.

4: Set $\widetilde{\widehat{f}}(\alpha)=0$ for all $\alpha \notin \alpha\left(\mathcal{L}^{\prime}\right)$.

5: Accept if there is a nonsingular linear transformation $A$ and $g \in \mathcal{G}$ such that $\sum_{\beta \in \mathbb{F}_{2}^{n}} \widehat{\widehat{f \circ A}}(\beta) \widehat{g}(\beta) \geq 1-\epsilon$, and reject otherwise.

to compute $\sum_{\beta \in \mathbb{F}_{2}^{n}} \widetilde{\widetilde{f \circ A}}(\beta) \widehat{g}(\beta)$, but by construction, $\widetilde{\widehat{f \circ A}}(\beta)=0$ when $\beta$ is not in $\operatorname{span}\left(\left\{\alpha\left(C_{1}\right), \alpha\left(C_{2}\right), \ldots, \alpha\left(C_{k}\right)\right\}\right)$. It is important to note that none of these steps require any further queries to $f$.

\section{Testing any linear-invariant property well-approximated by low spectral norm functions}

In this section, we prove Theorem 4 . Recall that we say that a property $P$ is linear-invariant if for every function $g$ that satisfies $P$ and every square matrix $A$, the function $g \circ A$ also satisfies $P$, where $(g \circ A)(x)=$ $g(A x)$ for every $x \in \mathbb{F}_{2}^{n}$. Our definition of linear isomorphism is with respect to nonsingular linear transformations.

In the implicit learning setting of [14] and [13], the algorithm uses enough examples to confidently check if the unknown function has a special structure. In the majority of cases, the algorithm simply uses brute force to check the consistency of the examples with a function satisfying the desired property. Thus, the query complexity depends on how many different functions the algorithm uses in its consistency-checking.

Our implicit learning algorithm behaves slightly differently. The algorithm uses Implicit Sieve to implicitly find heavy Fourier coefficients, then uses these estimates of these to form a multilinear polynomial that acts as an approximation of $f$. However, the algorithm does not check consistency with $g$. In fact, the entire algorithm and the analysis save for the last step do not depend on $g$ ! Our algorithm simply outputs some sort of hypothesis for $f$, which requires no queries to evaluate. Thus, we can test isomorphism to multiple functions with no effect on the query complexity of the algorithm. This modification is outlined in Algorithm 3. 
For a class of functions $\mathcal{G}$, we define the linear closure $L(\mathcal{G})$ of $\mathcal{G}$ to be $L(\mathcal{G})=\{g \circ A \mid g \in \mathcal{G}, A$ is a non-singular matrix $\}$. The previous iteration of our algorithm considered the case where $\mathcal{G}$ consists of a single function.

We could simply run the previous Algorithm 2 once save for the last step. On the last step, we try not only every nonsingular linear transformation applied to $f$, but also every function $g \in \mathcal{G}$. If one of the tests accepts, then $f$ is $\left(\frac{\epsilon}{3}, g\right)$-isomorphic. Thus, $f$ is $\frac{2 \epsilon}{3}$-close to $g$, and it follows that $f$ is $\frac{2 \epsilon}{3}$-close to $\mathcal{G}$. If $f$ is $\epsilon$-far from every function in $\mathcal{G}$, then $f$ is $\frac{2 \epsilon}{3}$-far from every function in $\mathcal{G}$. The failure probability is still just $\frac{7}{100}$, since the only failure probability comes from incorrect estimations of $f$. Thus, we get the following stronger version of our main theorem:

Theorem 6. Let $\mathcal{G}$ be a set of functions such that every function $g \in \mathcal{G}$ is $\frac{\epsilon}{3}$-close to having $\widehat{\|}_{\|} \widehat{\|}_{1} \leq s$. Then we can $\epsilon$-test membership in $L(\mathcal{G})$ with query complexity $\operatorname{poly}\left(s, \frac{1}{\epsilon}\right)$ queries.

Proof. We can exhaustively test $g$-isomorphism to every $g \in \mathcal{G}$. Because $\mathcal{G}$ is closed under nonsingular linear transformations, any function $h$ such that $h$ is $g$-isomorphic for some $g \in G$ is in $\mathcal{G}$. The result follows from the above argument along with Claims 13 and 14.

Further, we prove Theorem 4. Analogously to the definition of linear closure already given, given a set of functions $\mathcal{G}$, define $L^{\prime}(\mathcal{G})=\left\{g \circ A^{\prime} \mid\right.$ $g \in \mathcal{G}$ and $A^{\prime}$ is a linear transformation $\}$. It is easy to see that $L^{\prime}(\mathcal{G})$ is a linear-invariant property for any $\mathcal{G}$.

Theorem 7. Let $\mathcal{G}$ be any set of functions such that $\widehat{\|}_{\|} \widehat{\|}_{1} \leq s$ for all $g \in \mathcal{G}$. Then we can $\epsilon$-test membership in $L^{\prime}(\mathcal{G})$ with poly $(s, 1 / \epsilon)$ queries.

Proof. It is known that $\widehat{\|} g \widehat{\|}_{1} \geq \widehat{\|} g \circ A \widehat{\|}_{1}$ for any linear transformation $A$. Theorem 1 applies because $\widehat{\|} g \|_{1} \leq s$ for every function in $L^{\prime}(\mathcal{G})$. Thus, we can $\epsilon$-test $g$-isomorphism to every function in $L^{\prime}(\mathcal{G})$.

Theorem 4 follows since $P=L^{\prime}(P)$ for a linear-invariant property, so we can take $\mathcal{G}=P=L^{\prime}(\mathcal{G})$ assuming $P$ is linear-invariant.

Lemma 16. Let $g: \mathbb{F}_{2}^{n} \rightarrow\{-1,1\}$ be a function with $\widehat{\|}_{\|} \widehat{\|}_{1} \leq s$. We can $\epsilon$-test "g-homomorphism"; that is, we can $\epsilon$-test whether $f=g \circ A$ for some linear transformation.

Proof. Test isomorphism to every function in $L^{\prime}(\{g\})$.

Using the fact that every $r$-dimensional function has spectral norm at most $2^{r / 2}$, we get: 
Corollary 1. Suppose $\mathcal{G}$ is a class of functions such that every function in $\mathcal{G}$ is $\frac{\epsilon}{3}$-close to a function on $r(\epsilon)$ variables for every $\epsilon>0$. Then membership in $L(\mathcal{G})$ is $\epsilon$-testable with poly $\left(2^{r(\epsilon / 3)}, \frac{1}{\epsilon}\right)$ queries.

Thus, we have a constant-query tester for the linear closure of many natural properties. Each of these function classes is the natural extension replacing each variable with a parity of any length. As examples, we have the following:

Proposition 1. We can test the linear closure of:

- decision lists (using poly $(1 / \epsilon)$ queries.)

- decision trees of size $s$ (using poly $(s, 1 / \epsilon)$ queries.)

- DNF formulas with $s$ terms (using poly $\left(2^{s \log (s / \epsilon)}\right)$ queries.)

- Boolean formulas of size $s$ (using poly $\left(2^{s \log (s / \epsilon)}\right)$ queries.)

- Boolean circuits of size $s$ (using poly $\left(2^{s \log (s / \epsilon)}\right)$ queries.)

Proof. From [14], every decision list is $\epsilon / 3$-close to a function over $O(\log (1 / \epsilon))$ variables. It is well-known from [15] that if $g$ is computable by a decision tree with $s$ nodes then $\widehat{\|} g \widehat{\|}_{1} \leq s$, so we can apply Theorem 1 . For each of the last three, we apply the result of [14] that any function in each of the last three classes mentioned is $\epsilon / 3$-close to a function on $O\left(s \log \frac{s}{\epsilon}\right)$ variables.

\section{E Lower Bounds for Testing Isomorphism to Functions with Small Spectral Norm}

In this section we prove Theorems 2 and 3 . Theorem 2 can be easily obtained from the following lemma.

Lemma 17 ([19]). Let $g$ be a function $\epsilon$-far from having Fourier dimension at most $k$. Then, $\epsilon$-testing g-isomorphism requires $k-1$ queries.

Proof (of Theorem 2). Let $g$ be a function $\epsilon$-far from having spectral norm at most $s$. Then, it is easy to see that $g$ is $\epsilon$-far from being $s$-sparse, and it follows that $g$ is $\epsilon$-far from being ( $\log s$ )-dimensional. Then, we need at least $\Omega(\log s)$ queries to test $g$-isomorphism from Lemma 17 .

Now, we turn to showing Theorem 3. Let $T$ be a decision tree with the following properties: (1) $T$ forms a complete binary tree of depth $h$, where $h$ is a parameter chosen later, (2) every internal node uses different coordinate, and (3) for each deepest internal node using coordinate $x_{i}, T$ returns $c$ if $x_{i}=c$. The number of leaves in the decision tree is $2^{h}$. Let $g_{T}: \mathbb{F}_{2}^{n} \rightarrow\{-1,1\}$ be the function corresponding to $T$, where $n \gg 2^{h}$. Then, $g_{T}$ has the following properties. 
Lemma 18. The spectral norm of $g_{T}$ is at most $2^{h}$. Moreover $g_{T}$ is $\frac{1}{2^{h}}$ far from $2^{h-2}$-dimensional functions.

Proof. [16] showed that a function computed by a decision tree with $\ell$ leaves has spectral norm at most $\ell$. Thus, the first claim holds.

We now see the second claim. Let $L$ be the set of coordinates used by internal nodes of depth $h-1$, that is, deepest internal nodes. Then, for each $i \in L$,

$$
\begin{aligned}
& \widehat{g_{T}}(\{i\})=\mathbf{E}\left[g_{T}(\boldsymbol{x}) \boldsymbol{x}_{i}\right] \\
=\operatorname{Pr}[ & \left.T \text { does not use } \boldsymbol{x}_{i}\right] \cdot \mathbf{E}\left[g_{T}(\boldsymbol{x}) \boldsymbol{x}_{i} \mid T \text { does not use } \boldsymbol{x}_{i}\right] \\
& \quad+\operatorname{Pr}\left[T \text { uses } \boldsymbol{x}_{i}\right] \cdot \mathbf{E}\left[g_{T}(\boldsymbol{x}) \boldsymbol{x}_{i} \mid T \text { uses } \boldsymbol{x}_{i}\right] \\
= & \operatorname{Pr}[\boldsymbol{x} \text { uses } v] \cdot \mathbf{E}\left[g_{T}(\boldsymbol{x}) \boldsymbol{x}_{i} \mid T \text { uses } \boldsymbol{x}_{i}\right]=\frac{1}{2^{h-1}} .
\end{aligned}
$$

Let $g$ be the $2^{h-2}$-dimensional function closest to $g_{T}$, and let $S=\{i \mid$ $\widehat{g}(\{i\}) \neq 0\}$. Note that $|S| \leq 2^{h-2}$. However,

$$
\begin{aligned}
\operatorname{dist}\left(g_{T}, g\right) & =\frac{1}{4}\left\|g_{T}-g\right\|_{2}^{2} \geq \frac{1}{4} \sum_{i \in L \backslash S}\left|\widehat{g_{T}}(\{i\})-\widehat{g}(\{i\})\right|^{2} \\
& \geq\left(2^{h-1}-2^{h-2}\right) \cdot \frac{1}{2^{2(h-1)}}=\frac{1}{2^{h}}
\end{aligned}
$$

Proof (of Theorem 3). We set $h=\log \frac{1}{\epsilon}$. Then, $g_{T}$ has spectral norm at most $\frac{1}{\epsilon}$ whereas $g_{T}$ is $\epsilon$-far from every $\frac{1}{4 \epsilon}$-dimensional function. From Lemma 17, any $\epsilon$-tester for $g_{T}$-isomorphism requires $\Omega\left(\frac{1}{\epsilon}\right)$ queries. Thus, we have the desired lower bound. 